Conclusion: Combined, our findings suggest that the $\mathrm{CD} 4^{+} \mathrm{CD} 161^{+} \mathrm{C}$ $\mathrm{CR}^{+}{ }^{+} \mathrm{CR} 5^{+} \mathrm{T}$ cell subset represents a substantially abnormal $\mathrm{T}$ cell subset in $R A$, exhibiting exaggerated pro-inflammatory responses, numerical abundance relative to Tregs, and resistant to regulation by Tregs. The $\mathrm{CD} 4^{+} \mathrm{CD} 161^{+} \mathrm{C}$ $\mathrm{CR} 2^{+} \mathrm{CCR} 5^{+} \mathrm{T}$ cell subset appears to be a marker of therapeutic response status in RA, via its contribution to disease pathology and highlights this subset as a potential therapeutic target in RA.

References:

[1] Mclnnes IB, Schett G. The pathogenesis of rheumatoid arthritis. $N$ Engl J Med. 2011;365(23):2205-19.

[2] Mexhitaj I, Nyirenda MH, Li R, O’Mahony J, Rezk A, Rozenberg A, et al. Abnormal effector and regulatory $T$ cell subsets in paediatric-onset multiple sclerosis. Brain. 2019;142(3):617-32.

[3] Cosmi L, Cimaz R, Maggi L, Santarlasci V, Capone M, Borriello F, et al. Evidence of the transient nature of the Th17 phenotype of $\mathrm{CD} 4^{+} \mathrm{CD} 161^{+} \mathrm{T}$ cells in the synovial fluid of patients with juvenile idiopathic arthritis. Arthritis Rheum. 2011;63(8):2504-15.

Disclosure of Interests: Mukanthu Nyirenda: None declared, lain Mclnnes Grant/research support from: Bristol-Myers Squibb, Celgene, Eli Lilly and Company, Janssen, and UCB, Consultant of: AbbVie, Bristol-Myers Squibb, Celgene, Eli Lilly and Company, Gilead, Janssen, Novartis, Pfizer, and UCB, Carl Goodyear: None declared

DOI: 10.1136/annrheumdis-2020-eular.5525

\section{THU0040 $\quad$ PROTEINASE 3-REACTIVE B CELL RECONSTITUTION AFTER TREATMENT WITH RITUXIMAB FOR ANCA- ASSOCIATED VASCULITIS}

A. Berti ${ }^{1}$, S. Hillion ${ }^{2}$, A. Hummel ${ }^{3}$, E. Carmona ${ }^{3}$, T. Peikert ${ }^{3}$, C. Langford ${ }^{4}$, P. A. Merkel ${ }^{5}$, P. Monach ${ }^{6}$, P. Seo ${ }^{7}$, R. Spiera ${ }^{8}$, E. W. St. Clair ${ }^{9}$, F. Fervenza $^{3}$, K. Harris ${ }^{10}$, J. H. Stone ${ }^{11}$, J. O. Pers ${ }^{2}$, U. Specks ${ }^{3}$, D. Cornec ${ }^{2}$ on behalf of the RAVE group. ${ }^{1}$ Santa Chiara Hospital, Trento, Italy; ${ }^{2}$ Université de Bretagne Occidentale, Brest, France; ${ }^{3}$ Mayo Clinic, ROCHESTER, United States of America; ${ }^{4}$ Cleveland Clinic, Cleveland, United States of America; ${ }^{5}$ University of Pennsylvania, Philadelphia, United States of America; ${ }^{6}$ Brigham and Women's Hospital, Boston, United States of America; ${ }^{7}$ Johns Hopkins Medicine, Baltimore, United States of America; ${ }^{8} \mathrm{Hospital}$ for Special Surgery, New York, New York, United States of America; ${ }^{9}$ Duke University, Durham, United States of America; ${ }^{10}$ immune Tolerance Group, Bethesda, United States of America; ${ }^{11}$ Massachusetts General Hospital, Boston, United States of America

Background: Proteinase 3 (PR3)-reactive B cells are present in PR3-ANCA-associated vasculitis (AAV) at levels higher than healthy controls.

Objectives: To evaluate the dynamics of the PR3-reactive B cell repopulation in patients with PR3-AAV after treatment with rituximab, and to analyze possible associations between these immunological changes and long-lasting remissions.

Methods: We analyzed all available frozen peripheral blood mononuclear cells $(n=148$ ) from 23 randomly-selected PR3-AAV patients who participated in the RAVE trial and achieved complete remission (BVAS $=0$, prednisone $=0$ ) after treatment with rituximab.

We measured PR3-reactive $B$ cells and the relative subsets by a multi-color flow cytometry panel including CD19, IgD, CD27, CD38, CD24, and a biotinylated PR3 revealed by fluorescent streptavidin. The clinical data of the trial were correlated with flow-cytometry data.

Results: 10/23 (43\%) patients relapsed during the follow up, 8/10 relapses were severe. At baseline, clinical features, PR3-ANCA levels, \% of total PR3-reactive $B$ cells and PR3-reactive B cell subsets were similar between relapsers and non-relapsers. All patients were followed until the end of the trial, for a mean of 44 months (25-75\%IQR 31-54), without difference in follow-up time between relapsers and non-relapsers $(p=0.98)$.

The majority of patients had B cell repopulation at 12 (range 12-24) months after rituximab. At the time of $\mathrm{B}$ cell repopulation, transitional (CD19+CD24+CD38+) and naïve (CD19+CD27+lgD-) B cells were higher compared to baseline, while total plasmablasts (PB) were unchanged, and mature B cells significantly decreased in both relapsers and non relapsers. PR3-reactive $B$ cells reappeared in all the patients, and the \% of PR3-reactive of B cells were higher at the B cell repopulation visit compared to baseline $(5.82 \%$ vs $4.25 \%, p<0.05)$, while total $B$ cells were lower $(66 / \mu \mathrm{L}$ vs $151 / \mu \mathrm{L}, \mathrm{p}<0.01)$, regardless of future relapse.

Within PR3-reactive B cells, only the \% of PB (CD19+CD27+CD38+PR3+) were higher in relapsers vs. non-relapsers (median [25-75\%IQR]; 1.95\% [1.315-3.845] vs $0.84 \%$ [0.05-1.66], $\mathrm{p}=0.022$ ) and severe relapsers vs non-severe relapsers (2.165\% [1.66-4.315] vs $0.84 \%$ [0.1-1.74], $p=0.015)$. Time-to-relapse and time-to severe-relapse were significantly shorter in patients with circulating PR3-PB higher than the median value of the cohort $(1.6 \%)$ during $B$ cell reconstitution (Figure 1A-B).
Conclusion: In PR3-AAV, during $B$ cell reconstitution after rituximab, the total fraction of PR3-B cells increases, due to the expansion of the transitional and naïve B cell compartments. Circulating PR3-PB within PR3-B cells are enriched in the peripheral blood of relapsing and severely relapsing patients compared to non-relapsing patients. Higher levels of PR3-PB after rituximab during B cell reappearance significantly increased the risk of subsequent relapse and severe relapse.

References:

[1] Cornec D, Berti A, Hummel A, et al. J Autoimmun. 2017
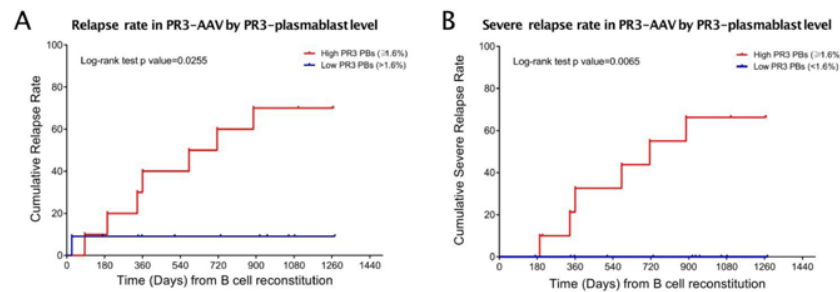

Disclosure of Interests: Alvise Berti: None declared, Sophie Hillion: None declared, Amber Hummel: None declared, Eva Carmona: None declared, Tobias Peikert: None declared, Carol Langford: None declared, Peter A. Merkel: None declared, Paul Monach: None declared, Philip Seo: None declared, Robert Spiera Grant/research support from: Roche-Genetech, GSK, Boehringer Ingelheim, Chemocentryx, Corbus, Forbius, Sanofi, Inflarx, Consultant of: Roche-Genetech, GSK, CSL Behring, Sanofi, Janssen, Chemocentryx, Forbius, Mistubishi Tanabe, E. William St. Clair: None declared, Fernando Fervenza: None declared, Kristina Harris: None declared John H. Stone Grant/research support from: Roche, Consultant of: Roche, Jacques-Olivier Pers: None declared, Ulrich Specks: None declared, Divi Cornec: None declared

DOI: 10.1136/annrheumdis-2020-eular.5169

\section{THU0041 IFNA AND IL21 PROMOTE DISTINCT POPULATIONS OF EFFECTOR B CELLS}

M. Boudigou ${ }^{1}$, A. Grasseau ${ }^{1}$, N. Chriti ${ }^{1}$, J. O. Pers ${ }^{1}$, L. Le Pottier ${ }^{1}$, S. Hillion ${ }^{1}$ ${ }^{1}$ Univ Brest, Inserm, LBAI, UMR1227, BREST, France

Background: B cells play a crucial role in the pathogenesis of systemic autoimmunity through various effector functions, including auto-antibody production secretion of pro-inflammatory cytokines and antigen presentation to $T$ cells. Interferon alpha (IFNa), mainly produced by innate cells (Menon et al., 2016) and interleukin (IL)-21 which is secreted by follicular helper T cells (Berglund et al., 2013), promote the generation of auto-reactive IgG-secreting plasma cells. However, it is suggested that IFNa participate also to the generation of regulatory B cells.

Objectives: To further understand the disturbing microenvironmental signals leading to autoimmune diseases, we aim to define a coherent framework integrating the B-cell subsets having different ability to respond to microenvironmental signals, and the signalling pathways driving the differentiation and the functional fate of $B$ cells.

Methods: Naïve and several populations of memory B cells were isolated from peripheral blood of healthy donors and differentiated in vitro in the presence of IL21 or IFNa. The phenotype and the expression of transcription factors were analysed by flow cytometry and molecular identity of these cells was further determined by transcriptomic approaches. Functional analyses were performed to assess the effector functions of $B$ cells and their potential regulatory effects on T cells.

Results: IFNa, in synergy with $\mathrm{CpG}$, promote the generation of CD27 $7^{\text {high }}$ CD38 ${ }^{\text {high }}$ plasmablasts (PB), mainly arising from memory subsets, but not IL21. However, IFNa and IL21 induce the transcriptional program of B-cell differentiation by up-regulating IRF4 and Blimp1 expression. Unlike IFNa, IL21 drive the

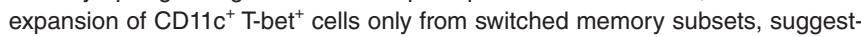
ing T-bet may be a footprint of long-lived memory B cells.

Even though subtle differences were observed in the antibody production between IFNa and IL21-stimulated memory B cells, naïve cells secreted less amount of IgM and IL10 when stimulated with IL21.

Transcriptomic studies are still in progress to further define the molecular profile of those distinct effector B cells.

Conclusion: Taken together, these findings suggest that IFNa promote a rapid differentiation of B cells into IL10 and Ig-secreting PB whereas IL21 contributes to the generation of T-bet ${ }^{+}$atypical pre-PB that may have a role in chronic autoimmune disorders. 


\section{References:}

[1] Berglund, L.J., Avery, D.T., Ma, C.S., Moens, L., Deenick, E.K., Bustamante, J., Boisson-Dupuis, S., Wong, M., Adelstein, S., Arkwright, P.D., et al. (2013) IL-21 signalling via STAT3 primes human naive B cells to respond to IL-2 to enhance their differentiation into plasmablasts. Blood 122, 3940-3950.

[2] Menon, M., Blair, P.A., Isenberg, D.A., and Mauri, C. (2016). A Regulatory Feedback between Plasmacytoid Dendritic Cells and Regulatory B Cells Is Aberrant in Systemic Lupus Erythematosus. Immunity 44, 683-697.

Disclosure of Interests: None declared

DOI: 10.1136/annrheumdis-2020-eular.5752

\section{THU0042 DIFFERENTIAL EFFECT OF ABATACEPT VS TNF BLOCKERS, ON THE FREQUENCY OF CIRCULATING FOLLICULAR HELPER (TFH) AND PERIPERAL HELPER (TPH) T CELLS IN RHEUMATOID ARTHRITIS}

P. Fortea-Gordo ${ }^{1,2}$, D. Peiteado ${ }^{1,2}$, A. Villalba ${ }^{1,2}$, M. J. Santos-Bornez ${ }^{1,2}$, L. Nuño ${ }^{1,2}$, I. Monjo ${ }^{1,2}$, A. Balsa ${ }^{1,2}$, M. E. Miranda-Carus ${ }^{1,2} .{ }^{1}$ University Hospital La Paz, Rheumatology, Madrid, Spain; ${ }^{2}$ IdiPAZ, Madrid, Spain

Background: CXCR5+PD- $1^{\text {hi }}$ follicular helper (Tfh) and CXCR5-PD-1 ${ }^{\text {hi }}$ peripheral helper (Tph) T cells play an important role in the pathogenesis of Rheumatoid Arthritis (RA) by providing help to autoantibody secreting B cells. Whereas Tfh cells typically dwell in the germinal centers of lymphoid organs, Tph cells accumulate at inflamed tissues. An increased frequency of Tph cells and of circulating counterparts of Tfh cells have been described in the peripheral blood of patients with seropositive RA.

Objectives: To examine the effect of treatment escalation using biological agents (TNF blockers or abatacept), on the frequency of circulating Tfh (cTfh) and Tph (cTph) cells in RA.

Methods: Peripheral blood was drawn from seropositive RA patients with an incomplete response to csDMARDS $(n=29)$ who initiated biological therapy with TNF blockers (TNFb) $(n=17)$ or abatacept $(n=12)$, prescribed based on routine clinical practice. cTfh and cTph cell frequencies were determined by flow cytometry of freshly isolated PBMCs at the basal visit and 6 months after starting treatment escalation. For each patient, an age and gender-matched healthy contro $(\mathrm{HC})$ was also studied at both time points $(n=29)$

Results: As compared with HC, active RA patients receiving csDMARDs demonstrated a baseline increased frequency of both cTfh and cTph cells. A significant improvement of disease activity as determined by the DAS28 score ( $\triangle \mathrm{DAS} 28>2.0$ ) was apparent in all of the patients 6 months after initiating biologicals. At that time point, a significant reduction of the previously elevated cTph cell frequency was observed in both treatment groups. However, cTfh cells remained elevated in patients receiving TNFb notwithstanding a good therapeutic response, whereas subjects receiving abatacept experienced a significant abatement of their cTfh cell frequency. Experimental variation of the cTfh and cTph cell numbers in $\mathrm{HC}$ was minimal.

Conclusion: Abatacept but not TNFb, are able to bring down cTfh cell numbers in RA. This indicates that costimulation blockade can help attain an immunological remission, whereas TNF neutralization may allow a persistent pathogenic germinal center overactivity. At the same time, treatment with both abatacept and TNF blockers results in a downmodulation of the previouly elevated cTph cell numbers, in parallel with the remitting local joint inflammation.

References:

[1] Simpson N et al, Arthritis Rheum 2010; Craft J, Nat Rev Rheumatol 2012;

Arroyo-Villa I et al., Arthritis Res Ther 2014; Rao DA et al., Nature 2017.

Disclosure of Interests: Paula Fortea-Gordo Grant/research support from: BMS, Diana Peiteado: None declared, Alejandro Villalba: None declared, Maria-Jose Santos-Bornez Grant/research support from: BMS, Laura Nuño: None declared, Irene Monjo: None declared, Alejandro Balsa Grant/research support from: BMS, Roche, Consultant of: AbbVie, Gilead, Lilly, Pfizer, UCB, Sanofi, Sandoz, Speakers bureau: AbbVie, Lilly, Sanofi, Novartis, Pfizer, UCB, Roche, Nordic, Sandoz, Maria-Eugenia Miranda-Carus Grant/research support from: BMS, Roche

DOI: 10.1136/annrheumdis-2020-eular.1823

\section{THU0043 EXPRESSION OF B CELL CHEMOKINE-CHEMOKINE RECEPTOR PATHWAYS IS ALTERED IN GIANT CELL ARTERITIS}

J. Graver ${ }^{1}$, A. Boots ${ }^{1}$, W. Abdulahad ${ }^{1}$, J. Bijzet ${ }^{1}$, D. Wolbers ${ }^{1}$, E. Brouwer ${ }^{1}$ M. Sandovici' . 'University Medical Center Groningen, Groningen, Netherlands

Background: The presence of organised B cells in both cranial-giant cell arteritis (C-GCA) (temporal artery) and large vessel (LV)-GCA (aorta) has previously been documented. The number and the extent of organisation of $B$ cells in tertiary lymphoid organs (TLO) was more prominent in the aorta than in the temporal artery, suggesting possible differences in B cell phenotype, kinetics and tropism between C-GCA and LV-GCA

Objectives: We sought to analyse B cell differentiation subsets in both C-GCA and LV-GCA and to investigate differences in the expression of chemokine pathways involved in B cell migration and TLO organisation.

Methods: Blood was collected from C-GCA $(n=11)$ and LV-GCA $(n=22)$ patients at baseline, before start of glucocorticoid treatment, and after 3 months of treatment. The LV-GCA groups consisted of 11 patients with isolated LV-GCA and 11 patients with overlap LV/C-GCA. Also, age- and sex- matched healthy controls $(\mathrm{HC}, \mathrm{n}=24)$ were included. The following chemokines were measured with Luminex in the sera of patients and HC: BAFF, CCL19, CCL21, CXCL9, CXCL10, CXCL11, CXCL12, and CXCL13. Thawed PBMC of 7 C-GCA, 10 LV-GCA and 24 $\mathrm{HC}$ were stained with antibodies against CD19, CD27, IgD, IgM, CD38, CXCR3 CXCR4, CXCR5, and CCR7 to allow identification of B cell differentiation subsets and their chemokine receptor expression.

Results: We found a lower absolute number of CXCR3+ memory and double negative (late stage) B cells in GCA patients when compared to healthy controls Also, the absolute number of CXCR5+ memory B cells was lower in patients than in controls. Chemokine receptor expression on circulating $B$ cells did not significantly differ between C-GCA and LV-GCA at baseline. After 3 months of treatment, frequencies and absolute numbers of both $\mathrm{CXCR} 3+$ and $\mathrm{CXCR} 5+$ memory B cells increased. In sera of all GCA patients, CXCL9 (which is a chemokine involved in migration of $B$ cells to sites of inflammation) and CXCL13 (which is involved in local organization of $B$ cells) were significantly increased. BAFF and CCL21 were increased only in LV-GCA when compared to HC. Serum chemokine levels did not differ between C-GCA and LV-GCA patients. An inverse correlation was observed between B cell counts and CXCL9 as well as CXCL13 in LV-GCA, only. After 3 months of treatment, CXCL9 levels remained elevated whereas CXCL13 increased even further.

Conclusion: At diagnosis, CXCL9 and CXCL13 were significantly increased in all GCA patients as compared to HC. Elevated CXCL9 levels inversely correlated with $B$ cells numbers in LV-GCA, only, which may suggest that $B$ cells preferentially migrate to the inflamed aorta via a mechanism involving CXCL9. In addition, CXCL13 may be linked to local TLO organization in LV-GCA. Currently, we are studying the local expression of chemokines and chemokine receptors at the site of inflammation in both C- and LV-GCA.

Disclosure of Interests: Jacoba Graver: None declared, Annemieke Boots Consultant of: Grünenthal Gmbh until 2017, Wayel Abdulahad: None declared Johan Bijzet: None declared, Daphne Wolbers: None declared, Elisabeth Brouwer Consultant of: Roche (consultancy fee 2017 and 2018 paid to the UMCG), Speakers bureau: Roche (2017 and 2018 paid to the UMCG), Maria Sandovici: None declared

DOI: 10.1136/annrheumdis-2020-eular.4435

\begin{tabular}{|l|l|}
\hline THU0044 & SINGLE CELL ANALYSIS OF BONE MARROW AND \\
& PERIPHERAL ALTERED B CELL DIFFERENTIATION \\
& IN PATIENTS WITH ACTIVE SLE AND THE \\
& MECHANISM OF ABNORMAL EARLY B CELL \\
& DEVELOPMENT
\end{tabular}

T. Fu ${ }^{1}$, Y. Yang ${ }^{1}$, X. Gu${ }^{1}$, C. Dong ${ }^{1}$, R. Zhao ${ }^{1}, \mathrm{~J} \mathrm{Ji}^{1}, \mathrm{X}$. Zhang $^{2}$, Z. Gu ${ }^{1} .{ }^{1}$ Affiliated Hospital of Nantong University, Nantong, China; ${ }^{2}$ Institute Pasteur of Shanghai Chinese Academy of Sciences, Shanghai, China

Background: B cell differentiation and dysfunction play a key role in the pathogenesis of Systemic lupus erythematosus (SLE). Bone marrow (BM) is the development organ of B cells, and also the home and residence place of plasma cells and memory B cells. However, there is a lack of studies on B cells in BM with lupus.

Objectives: To map the development of BM and peripheral B cells and investigate the mechanism of abnormal early $B$ cell development in SLE.

Methods: A total of 11 SLE patients and 5 age- and sex-matched controls were recruited.BM and peripheral $B$ cell subsets were measured by flow cytometry. sorting-purified B cell subsets were subject toSingle-cell RNA sequencing (scRNA-seq) and functional studies. Plasma cytokines and secreted immunoglobulins were detected by Luminex or ELISA. Disease activity of SLE patients was measured using the SLE Disease Activity Index (SLEDAI).

Results: In the present study, we find out that the percentage of monocytes in MNC $(p=0.070)$ and plasma cells $(p=0.001)$ in CD19+ were significantly decreased in BM of SLE, compared to healthy controls. While, SLE patients had increased $\mathrm{T} \% \mathrm{MNC}(\mathrm{p}=0.008)$ and $\mathrm{B} \% \mathrm{CD} 19+(\mathrm{p}=0.002)$ in $\mathrm{BM}$ that controls. In detail, the $B$ cell subsets of bone marrow in patients with active lupus (SLEDAI $\geq 8$ score) were seriously disordered, showing the increasing $\operatorname{ToMNC}(p=0.049)$ propre-B\%CD19+ $(p=0.006)$ and immature B cell\%CD19+ $(p=0.010)$ than healthy donors. propre-B\%CD19+ exhibited good relationship with SLEDAI. By 